\title{
The RoboPol Program: Optical Polarimetric Monitoring of Blazars
}

\author{
Dmitry Blinov 1,2,3,*(D) and Vasiliki Pavlidou 1,2,*(D) \\ 1 Physics Department \& Institute of Theoretical \& Computational Physics, University of Crete, \\ 71003 Heraklion, Crete, Greece \\ 2 IESL \& Institute of Astrophysics, Foundation for Research and Technology-Hellas, \\ 71110 Heraklion, Crete, Greece \\ 3 Astronomical Institute, Saint Petersburg State University, 198504 Saint Petersburg, Russia \\ * Correspondence: blinov@physics.uoc.gr (D.B.); pavlidou@physics.uoc.gr (V.P.)
}

Received: 28 February 2019; Accepted: 8 April 2019; Published: 10 April 2019

check for updates

\begin{abstract}
After three years of polarimetric monitoring of blazars, the RoboPol project has uncovered several key characteristics of polarimetric rotations in the optical for these most variable sources. The most important of these is that polarization properties of the synchrotron emission in the optical appear to be directly linked with gamma-ray activity. In this paper, we discuss the evidence for this connection, as well as the broader features of polarimetric behavior in blazars that are key in making progress with theoretical modeling of blazar emission.
\end{abstract}

Keywords: active galaxies; blazars; polarization; gamma rays; high energy; monitoring

\section{Introduction}

The first measurements of optical polarization of quasars followed soon after the identification of their optical counterparts. These measurements have revealed that many of these sources are linearly polarized to some extent, and variable in both the degree of polarization and the electric vector position angle (EVPA) [1]. Fifteen years later, optical polarization was measured for $\sim 60$ compact extragalactic sources, and the first statistical conclusions could be derived, together with some preliminary tests of consistency with models [2]. It was found that, typically, the behavior of polarization degrees and angles are erratic [3]. This can be explained by the presence of a persistent component and one or multiple variable components [4-7]. In fact, such a model was first used for the interpretation of irregular radio polarization behavior as early as 1967 [8]. However, it has been known since the end of the 1970s that some sources exhibit long monotonic rotations of the EVPA in the radio band emission during flares $[9,10]$. The first optical polarization rotation, simultaneous with a similar rotation in the radio band, was reported by Kikuchi et al. (1988) [11]. During the first years of operation of the Fermi $\gamma$-ray observatory, optical EVPA rotations have attracted a lot of interest, because it was noticed that some of the strong $\gamma$-ray flares were accompanied by these events [12-14]. If EVPA rotations were physically related to the high-energy activity, they could help localize and clarify the nature of the latter one, which was actively discussed at that time.

Therefore, a number of optical polarization monitoring programs have since focused on observations of blazars that are bright and active in $\gamma$-rays. Such major monitoring programs have been carried out by the Boston University group [15], at St. Petersburg University [16], at the Hiroshima University (Kanata program) [17], at the Steward Observatory [18], at the Calar Alto observatory [19], and at the Tuorla observatory [20].

From a theoretical standpoint, a wide variety of mechanisms have been proposed to explain the origin of EVPA rotations. They include the following scenarios: 
1. A two-component model, where changes in the polarization are due only to changes in the relative flux of the two components [21].

2. Propagation of an emission component along the jet with a non-axisymmetric magnetic field [22].

3. Polarized synchrotron flares rotating in the accretion disc [23].

4. Motion of an emission component along a helical streamline in the helical magnetic field [12,14].

5. Jet bending [13].

6. Changes of polarization parameters and total flux due to the Doppler factor variability [24].

7. Random variation of the polarization vector in the emission produced by a turbulent zone $[10,25,26]$.

8. Propagation of a relativistic shock in the jet that causes compression of the magnetic field and alters its direction and the degree of ordering [27].

9. Precession of the jet [28].

10. Kink instability [29].

11. Magnetic reconnections [30].

12. Relativistic EVPA rotation in the observer frame combined with the conical jet geometry and particle cooling [31].

Inspired by the perplexing and unclear nature of EVPA rotations, an intensive polarization monitoring program, RoboPol, was designed and executed.

\section{The RoboPol Program}

In spite of the immense significance of EVPA rotations for an understanding of the magnetic field structure in the jets and their importance for constraining theoretical models, only a handful of these events were reported in the 25 years since their discovery. Additionally, most of these observing programs were monitoring small numbers of the most violently variable blazars; these were biased samples, and not appropriate for statistical studies. Therefore, such questions as "Do all blazars rotate the EVPA? How often these rotations occur on average? Does this depend on the blazar class?" would be impossible to address accurately using data of monitoring programs already existing at the time when RoboPol was designed.

In order to overcome these shortcomings, an international collaboration between the University of Crete and Foundation of Research and Technology Hellas (FORTH, Greece), the Max-Planck Institute for Radioastronomy (Germany), the California Institute of Technology (USA), the Inter-University Centre for Astronomy and Astrophysics (India), and the Nicolaus Copernicus University (Poland) developed a new blazar monitoring program called RoboPol. This program specifically aimed at maximizing the number of detected EVPA rotations and characterizing the optical polarization behavior of blazars.

\subsection{Instrumentation}

The key element of the the RoboPol program is a novel-design optical polarimeter that was developed specifically for the project. This polarimeter has a fixed set of half wave plates and Wollaston prisms (see Figure 1) that split each incident ray into four rays with the polarization plane rotated by $45^{\circ}$ with respect to each other. Therefore, one can measure the Stokes parameters $q=Q / I$ and $u=U / I$ of the linearly polarized light in one exposure. Given the large field of view $\left(13^{\prime} \times 13^{\prime}\right)$, the total flux density $I$ can also be measured at the same time if a comparison star with a known magnitude is present in the field. Since the polarimeter has no moving parts except the filter, it avoids systematic uncertainties related to an imperfect alignment of rotating optical elements, and simplifies the instrument calibration.

The polarimeter was hosted on a $1.3 \mathrm{~m}$ telescope at the Skinakas Observatory, which is a joint facility of the FORTH and the University of Crete. It is located at an altitude of $1750 \mathrm{~m}$ in Crete $\left(35.2120^{\circ} \mathrm{N}, 24.8982^{\circ} \mathrm{E}\right)$. 


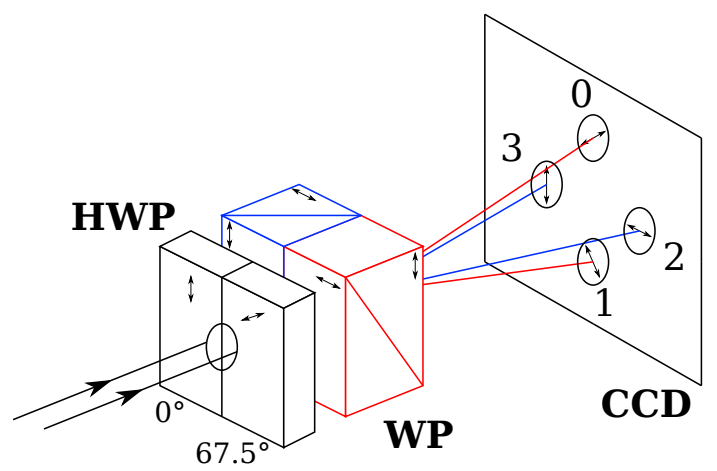

Figure 1. Schematic of the polarizing optics of RoboPol. The array of two half-wave plates (HWP) and two Wollaston prisms (WP) is shown. The fast optical axes of the elements and the polarization planes of the rays are shown with arrows. The HWP with the fast axis oriented at $67.5^{\circ}$ rotates the polarization plane by $2 \cdot 67.5=135^{\circ}$ (see, e.g., [32]).

The site offers seeing conditions which are among the best in Europe, comparable to several of the largest facilities in the world (median seeing of 0.7 arcsec [33]). The Skinakas Observatory granted the RoboPol collaboration four nights per week, on average, over the entire Skinakas observing season (May to November each year), for three years (2013-2015).

In addition to the advanced instrument, we developed an automated telescope control system, as well as a processing pipeline. The former one was able to point automatically and estimate the brightness of targets using the pointing exposures. It could also estimate polarization parameters on the fly, reaching a particular signal-to-noise ratio in EVPA if required.

A more detailed description of the polarimeter can be found in Ramaprakash et al. (2019) [34], while the pipeline and the control system are presented in detail in King et al. (2014) [35].

\subsection{Unbiased Observing Sample}

Another unique feature of the program is the observing sample, constructed in a way which allows for systematic statistical studies. We built the sample relying on unbiased and statistically rigorous criteria. Firstly, we constructed a flux-limited sample of $\gamma$-loud blazars from the second Fermi-LAT (2FGL) source catalog [36], selecting sources classified as BL Lac (bzb), FSRQ (bzq), or an active galaxy of uncertain type (agu) that have the integrated photon flux $F(100 \mathrm{MeV} \leq E \leq$ $100 \mathrm{GeV}) \geq 2 \times 10^{-8} \mathrm{ph} \mathrm{cm}^{-2} \mathrm{~s}^{-1}$. Then, we excluded sources with $|b| \leq 10^{\circ}$ in order to avoid any dependence of the sensitivity on the photon index and minimize polarization induced by the Galactic dust. Finally, applying visibility constraints and introducing the optical magnitude cut $R \leq 17.5$, we obtained the unbiased $\gamma$-loud monitoring sample of 62 sources. The control sample of $\gamma$-quiet sources was selected from Candidate Gamma-Ray Blazar Survey (CGRaBS) sources [37] that had not been detected by Fermi at that time (not included in 2FGL). We introduced constraints on the $15 \mathrm{GHz}$ mean flux density $\geq 0.06$ Jy and intrinsic modulation index as defined by Richards et al. [38] $m \geq 0.05$. Then, we applied similar visibility cuts and the $R \leq 17.5$ condition as for the $\gamma$-loud sources. Finally, we randomly selected 15 sources that passed all the cuts. Two out of 15 sources in our $\gamma$-quiet sample appeared in the four-year Fermi-LAT (3FGL) catalog as $\gamma$-ray sources. Therefore, we added two more blazars that satisfied the previous criteria, but were not reported as $\gamma$-ray-loud in either 2FGL or 3FGL. Additionally, to the unbiased samples of $\gamma$-loud and -quiet sources, we observed 24 additional "high interest" sources, which were not taken into account in population studies where they could bias results due to their activity being higher than average. More details on the sample selection are discussed in Pavlidou et al. (2014) [39]. 


\section{Results}

After running the program for three observing seasons, we identified 40 EVPA rotations in 24 individual blazars (see Section 4 for discussion on the EVPA rotation definition). As an example, in Figure 2 we show one of the rotation events observed in the $\gamma$-ray-loud sample blazar J1751+0939. We were able to study statistical properties of the detected events, their characteristics, and connection to the high-energy flares in blazars. Here, we briefly discuss our major results related to the EVPA rotations, as well as the behavior of polarization in blazars in general.
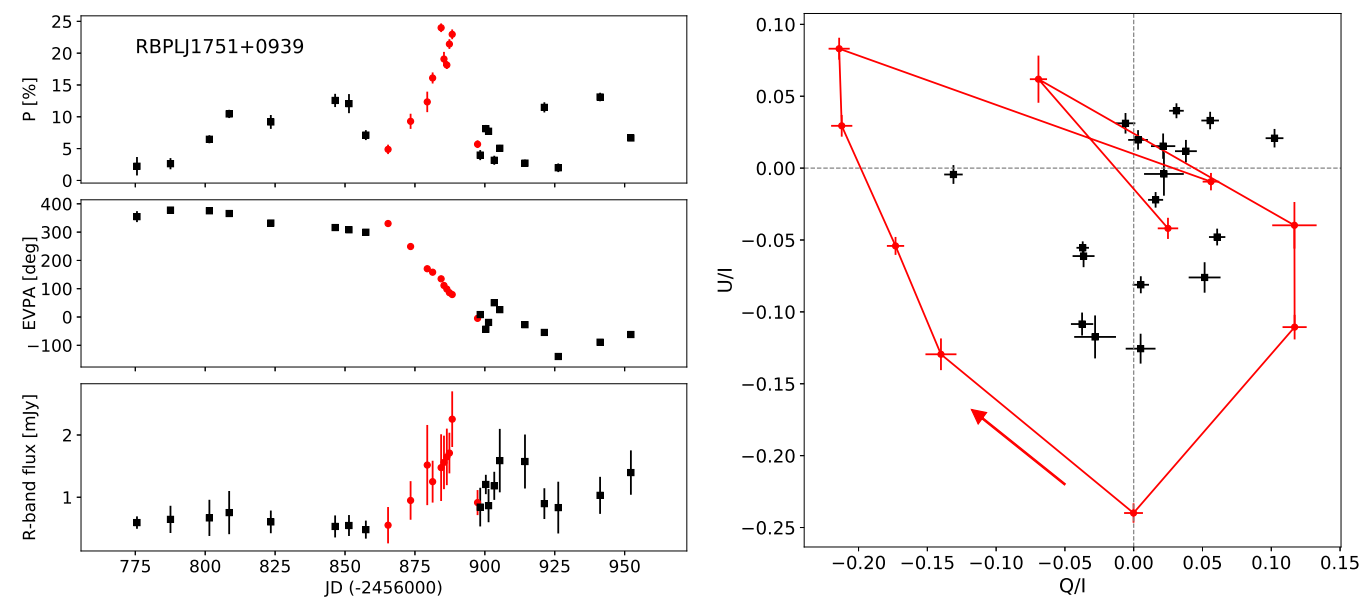

Figure 2. An example of an EVPA rotation (marked by the red circles). Based on data from [40].

\subsection{Polarization Properties of Different Types of Blazars}

Using data obtained during the first month of RoboPol operation, we have found that the average fractional polarizations of the $\gamma$-loud and $\gamma$-quiet samples are $\langle p\rangle=6.4_{-0.8}^{+0.9} \times 10^{-2}$ and 3.2 ${ }_{-1.1}^{+2.0} \times 10^{-2}$, respectively. The hypothesis that the polarization fractions of blazars in these samples are drawn from the same distribution is rejected at the $3 \sigma$ level of significance [39]. Later, this result was confirmed using averaged monitoring data for a larger sample of sources [41]. There, the difference of average intrinsic polarization for the two groups was found to be even larger, $9.2 \pm 0.8 \times 10^{-2}$ and $3.1 \pm 0.8 \times 10^{-2}$. A similar conclusion that $\gamma$-ray luminosity and optical polarization are correlated was made, also based on Kanata monitoring data [42]. These results immediately suggest that the average "polarizing efficiency" in jets of $\gamma$-loud and $\gamma$-quiet blazars is systematically different. This efficiency depends on the ordering of the magnetic field in the jet and the electron energy distribution index. However, the latter is a rather weak dependence, and cannot solely be responsible for the wide range of optical polarization observed in blazars (see discussion in [42]).

As per Hovatta et al. [43], we tested whether the BL Lac objects that were detected with the current-generation $\mathrm{TeV}$ instruments had different optical polarization characteristics compared to similar sources that have not yet been detected. It was found that after correction for depolarization produced by the host galaxies, TeV-detected and non-detected BL Lacs had the same mean fractional polarization. The rate of EVPA variations is also statistically indistinguishable between the two groups. This was considered as an indication that there are no intrinsic differences in the polarization properties of the TeV-detected and non-detected high-energy BL Lac objects. Therefore, the magnetic field ordering and the electrons distribution within their jets are not expected to differ systematically.

Besides the relation of optical polarization with $\gamma$-ray emission of blazars, it has also been found that polarization parameters depend on the position of the synchrotron peak maximum. For instance, low-synchrotron peaked (LSP, $\log \left(v_{p}\right)<14$ ) sources have higher fractional polarization and a broader range of values compared to intermediate- (ISP, $14 \leq \log \left(v_{p}\right) \leq 15$ ) and high-synchrotron peaked (HSP, $15<\log \left(v_{p}\right)$ ) sources [41]. The dependence of the maximum polarization on the synchrotron peak position was also found in other monitoring programs [42,44]. Moreover, HSP sources tend to 
demonstrate significant peaks in EVPA distributions (i.e., a preferred direction of polarization plane), while LSPs typically have uniform distributions of EVPA. Hints of this behavior were noted earlier, but for very small samples of sources so that no statistically robust conclusion could be made [7].

Considering the aforementioned dependencies, Angelakis et al. [41] proposed a qualitative model sketched in Figure 3. In this scenario, a mildly relativistic shock in the jet causes efficient particle acceleration (e.g., due to diffusive shock acceleration or magnetic reconnection) in a small volume directly downstream of the shock. Propagating further downstream, these accelerated particles progressively lose their energy due to the emission of inverse Compton and synchrotron radiation. Therefore, the emission at the synchrotron peak frequency or higher is produced by the most energetic particles congregated in a small volume adjacent to the shock, where the magnetic field is compressed and expected to be highly ordered. Lower-frequency synchrotron emission is received from a region with greater volume downstream the jet. This region contains a larger range of magnetic field line orientations. Additionally, the ordered component of the magnetic field can be less dominant in this zone, when compared to the turbulent component. Thus, at the fixed frequency of the R-band, we observe highly polarized emission near the synchrotron peak maximum and above in LSP sources, and significantly less polarized emission at the low-energy side of the peak in HSP blazars (see Figure 3). Itoh et al. [42] also explain the lower polarization of HSP sources by larger emitting volume and superposition of multiple emitting regions with independent orientations of magnetic field lines. However, their scenario includes a spine-sheath model, where the spine has a highly ordered magnetic field and produces highly polarized and variable emission, while the sheath, in contrast, produces steady and low-polarized light. Then, the systematic difference in the polarization of HSP and LSP sources can be caused by the increasing jet volume fraction occupied by the spine. Potentially, an observational test can discriminate between these two models. The first model predicts that most of the sources must demonstrate decreasing fractional polarization from higher (Ultraviolet) to lower frequencies (Near Infrared), while for the spine-sheath model, no systematic behavior is expected.

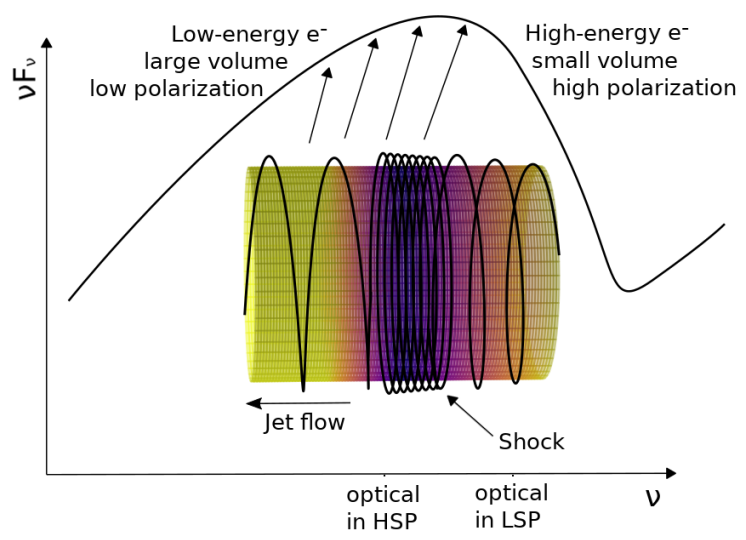

Figure 3. Sketch of the shock-in-jet scenario proposed by Angelakis et al. [41].

\subsection{Connection of EVPA Rotations and $\gamma$-Ray Flares}

One of the main goals of the project was to verify the previously assumed connection of EVPA rotations with strong $\gamma$-ray flares. We addressed this issue in [45] based on the first-season data and in [46] using the entire set of 40 events detected during the three-year program. Our main test included a direct estimation of the probability that all EVPA rotations occur at random times with respect to $\gamma$-ray flares in the light curves of corresponding blazars.

For this purpose, we identified all flares in $\gamma$-ray light curves that occurred during intervals covered by RoboPol. Then, we fitted these flares with an analytic function and derived their basic parameters, such as the peak time, flux at the peak, rise, and decay times. After this, we found the time lags between EVPA rotations and flares and constructed the cumulative distribution function (CDF) of 
their values (see Figure 4). Dropping random points at the $\gamma$-ray light curves, we ran a Monte-Carlo simulation that simulated EVPA rotations distributed at random moments in time. Thus, we obtained $10^{6}$ simulated CDFs ( $10^{4}$ of them are shown in Figure 4), and found that only 70 were entirely located closer to zero than the observed one. Therefore, we concluded that the probability of all time lags in the sample being accidentally so close to zero, as observed, is $\sim 7 \times 10^{-5}$. This implies that at least some of the time lags between EVPA rotations and $\gamma$-ray flare we observed are physically linked.

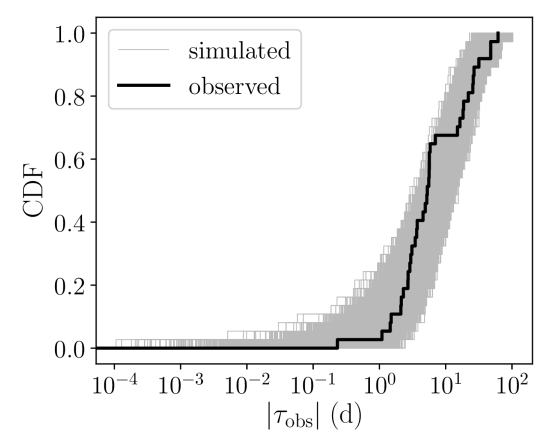

Figure 4. CDFs of the time lags $\tau_{o b s}$ between the EVPA rotations and peak moments of the closest $\gamma$-ray flares for the main sample rotators. The black line represents observed time lags, and the thin grey lines represent $10^{4}$ simulated values for the whole sample of rotations. Based on data from [46].

This interpretation received further support when we compared amplitudes and timescales of EVPA rotations and $\gamma$-ray flares (see Figure 5). In spite of large uncertainties in determination of the rotations amplitudes $\Delta \theta_{\max }$ (because of the difficulty in identifying the onset and the end of a particular event), it has been found that the peak luminosities of flares $L_{p}$ closest to rotations are anticorrelated with $\Delta \theta_{\max }$. Such a relation could be caused by a correlation of jet parameters that influence luminosity, i.e., the Lorentz and Doppler factors and the jet viewing angle, with the rotations amplitudes. The correlations between these parameters were indeed found to be significant as well. Moreover, we demonstrated that the characteristic timescales of rotations and $\gamma$-ray flares were marginally correlated [46]. Thus, one of the most significant outcomes of the RoboPol program is that it has proven, for the first time in a statistically robust fashion, that at least some EVPA rotations must be physically related to $\gamma$-ray flares.
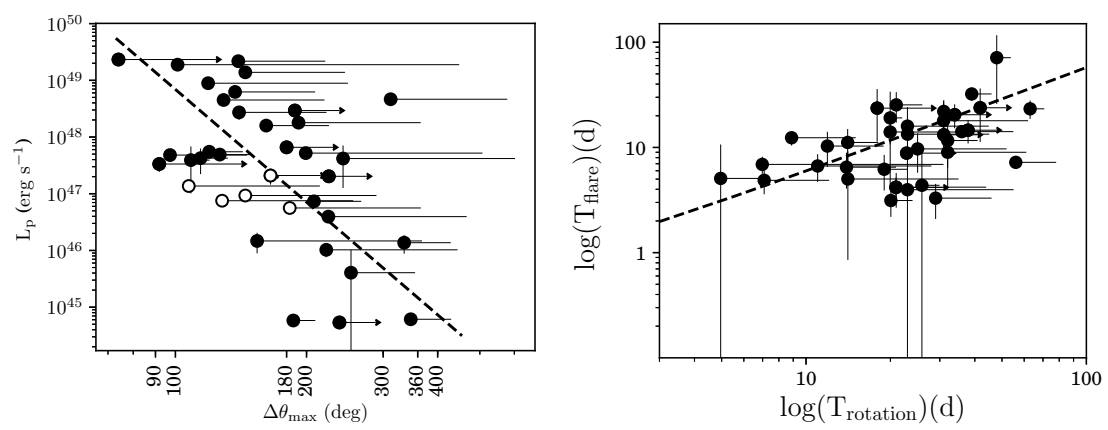

Figure 5. Left panel: $\gamma$-ray flare luminosity, $L_{p}$, vs. EVPA rotation amplitude, $\Delta \theta_{\max }$. Empty circles correspond to blazars with uncertain z. Right panel: characteristic timescale of flares, $T_{\text {flare }}$, vs. duration of rotations, $T_{\text {rotation }}$, in the observer frame Lines show linear fits to the data. Based on data from [46].

\subsection{Properties of EVPA Rotations}

RoboPol provided enough data for a first attempt to characterize the statistical properties of EVPA rotation events, as well as the behavior of the total flux and fractional polarization during these events. We detected EVPA rotations with durations, $\Delta T$ from 19 to 518 days in the jet reference frame and 
amplitudes, $\Delta \theta$ from $74^{\circ}$ to $427^{\circ}$ (see Figure 5). The histogram of rotation amplitudes was found to be consistent with both normal and uniform distributions. However, it has an apparent peak near the mean $186^{\circ}$ value, which is consistent with "the magic number" of $180^{\circ}$ appearing in several models explaining these events. When EVPA rotations are approximated by straight lines, their rotation rates $\Delta \theta / \Delta T$ in the jet reference frame span from $0.2^{\circ} / \mathrm{d}$ to $16^{\circ} / \mathrm{d}$. They are strongly non-uniform and, presumably, can be approximated by a power law distribution. This, however, could just be a natural outcome of $\Delta \theta$ and $\Delta T$ being uniformly distributed. The values of $\Delta \theta$ and $\Delta T$ are not correlated. We have presented statistical evidence that $\Delta \theta$ and $\Delta T$ of the rotations are likely to be limited-that is, arbitrary lasting and high amplitude events are not present in nature. The null hypotheses that rotation amplitudes and durations can exceed $460^{\circ}$ and 500 days are rejected at the significance level of 0.01 . These limits are presumably caused by restraints of the physical parameters presented in the jets, such as topology of the magnetic field, emission region size, and finite bulk speed of the moving emission features, or other characteristics related to the EVPA rotations.

We have demonstrated that most of the rotations are accompanied by a decrease in the fractional polarization. At the same time, its modulation indices, on average, remain constant. Moreover, we have found a marginal (2.7 $\sigma$ significance level) anticorrelation of the depolarization factor-that is, average polarization during rotation normalized by its value in a non-rotating state, with the rotation rate in the jet reference frame. This dependence, if confirmed with a larger sample of events, could place significant constraints on models explaining EVPA rotations. Finally, it has been demonstrated that during most of the EVPA rotations, there is no clear systematic increase in the total flux density. These and other properties have been reported in $[40,45-47]$.

\subsection{Rotations in Different Types of Blazars}

Thanks to the unbiased nature of the RoboPol monitoring sample, we were able to investigate the occurrence of EVPA rotations in blazars of different classes [47]. We started by demonstrating that the polarization plane rotations frequency (at least for events with rates $<20^{\circ} / d$ ) is not the same for different sources. For instance, the null hypothesis that EVPA rotations occur equally frequently in all sources was found to be very unlikely $\left(p=10^{-7}\right)$. At the same time, this difference in the EVPA rotations occurrence could not be clearly related to the $\gamma$-quiet - $\gamma$-loud dichotomy of blazars. We could not reject the null hypothesis that the $\gamma$-quiet sample blazars show rotations with the same frequency as sources in the $\gamma$-loud sample. Therefore, according to our data, it is not impossible that the frequency of EVPA rotations is the same for the blazars in the main and the control samples. Nevertheless, we found that the frequency of rotations very significantly varies, even between sources of the main $\gamma$-loud sample itself. For example, the upper limit on the average frequency of rotation in the $\gamma$-loud sample sources, where we detected zero rotations, is more than two orders of magnitude smaller than frequency of rotations in three sources which exhibited four rotations during the three years of monitoring. Furthermore, we verified that no external reason can entirely explain the distinction in rotation frequencies. A difference in accuracy of EVPA measurements that is caused by various fractional polarization levels cannot be solely responsible for this effect. Similarly, we have shown that there is no significant distinction in the time-delay factors $\delta /(1+z)$ between the samples with detected rotations and without. Therefore, we concluded that there exists a sub-class of objects that exhibit EVPA rotations significantly more frequently than others. This difference is not simply related to whether or not a blazar is detected by Fermi-LAT. It also cannot be explained by the non-uniformity of observations or by observational biases due to differences in the average fractional polarization [47].

We also looked for other intrinsic reasons that may cause the difference in rotation rates. We compared numbers of EVPA rotations and numbers of sources where these events occurred (rotators) for the three classes of blazars: low-synchrotron peaked (LSP: $\log \left(v_{p}\right)<14$ ), intermediate(ISP: $14 \leq \log \left(v_{p}\right)<15$ ), and high-synchrotron peaked (HSP: $15 \leq \log \left(v_{p}\right)$ ). It was found that rotations tend to occur in sources with a lower synchrotron peak (see Figure 6). For instance, the probability 
that sources with rotations were randomly drawn from the parent sample is $1.4 \%$ if both $\gamma$-quiet and $\gamma$-loud samples are considered as the parent sample. In the case when we consider only the $\gamma$-loud sample, this probability is reduced to $0.5 \%$. This tendency of EVPA rotations occurring in LSP blazars is in agreement with findings of Hovatta et al. [48], who has shown that LSP blazars are more variable than HSP in the total optical flux density (Stokes parameter I). Their work explains this finding with a scenario similar to the one sketched in Figure 3 and discussed before-that is, the LSP sources are observed near and above the electron energy peak, which causes stronger variations of the emission compared to HSP sources, where the lower energy electrons cool down slowly and produce mild variability. This scenario is expected to work for the polarized flux and EVPA variability as discussed in Section 3.1.

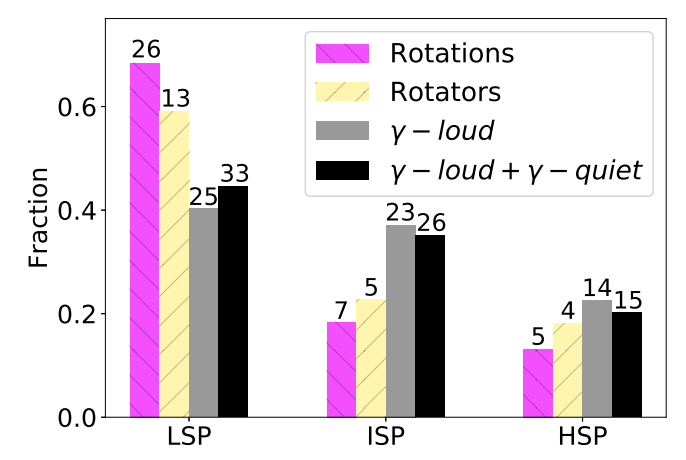

Figure 6. Occurrence of rotations and rotators (sources exhibiting rotations) among blazars of different synchrotron peak position class. Numbers correspond to the number of blazars (the number of events in the case of rotations) in each class. Based on data from [47].

We can briefly summarize the major results of the RoboPol project as follows: only about a quarter of blazars ( $\sim 28 \%$ of sources in both $\gamma$-loud and $\gamma$-quiet samples) exhibit EVPA rotations with rates $<20^{\circ} / \mathrm{d}$ in the optical band. The average frequency of appearance of these events is $1 / 232 \mathrm{~d}^{-1}$ (in the observer frame). The remaining part of sources $\sim 72 \%$ did not show any rotations registered in our data. Even if they do produce EVPA rotations, this should happen with average frequency less than $\sim 1 / 3230 \mathrm{~d}^{-1}$ in that sample.

\subsection{EVPA Rotations as Random Walks}

The EVPA behaviour in blazars most of the time is consistent with random walk variability [3]. This leads to the natural suspicion that long periods of monotonic change of the EVPA in one direction is nothing but a rare accidental outcome of the same random walk process. We tested the hypothesis that all EVPA rotations detected during the first observing season of RoboPol could be explained by stochastic variability of EVPA and found it to be unlikely [45]. In Kiehlmann et al. [49] a similarly simple random walk model was tested against EVPA variability in the three years of monitoring data. They found that the model is able to reproduce many parameters including durations, amplitudes and rates of EVPA rotations. However, it failed in simulating realistic distributions of the mean and standard deviation of the fractional polarization. This potentially could be corrected by more realistic models, such as by introduction of several populations of sources with different intrinsic variability properties. More comprehensive models that take into consideration both turbulent and ordered magnetic components as well as realistic electron energy and density distributions are successful in reproducing the evolution of polarization parameters and spectral energy distribution in high detail [25]. RoboPol data do not reject the hypothesis that individual EVPA rotations could be produced by a random walk of the polarization vector. Nevertheless, the correlations between parameters of EVPA rotations and $\gamma$-ray flares parameters described in Section 3.2 would be totally smeared out if all rotations were produced by random walks. Therefore, we deduced that at least some rotations are influenced by deterministic processes that define their properties. 
It is not impossible that both types (deterministic and stochastic) of EVPA rotation events coexist. Moreover, they may be present in the emission of a single blazar [26]. It could be that deterministic ones are produced during periods of high activity in the total flux and high polarization state. While random walks are more likely to detect during low polarization states, because the uncertainty of EVPA measurements is larger at these periods [50]. This would explain why, on average, we do not see an increase of optical total flux during rotations and observe depolarization during most of the events.

\subsection{Possible EVPA Rotations in NLSy1 Galaxies}

Radio loud narrow line Seyfert 1 galaxies are an enigmatic type of AGN that host relativistic jets and have been detected in $\gamma$-rays [51]. They, presumably, represent the low luminosity and the low black hole mass tail of the flat spectrum radio-quasars population [52]. Therefore, it is expected that their polarization properties may be similar to properties of LSP blazars, and they may exhibit high polarization variability together with EVPA rotations. Indeed, despite a lack of optical polarization measurements, at least some of NLSy1 have demonstrated high fractional polarization variability down to minute scales [53-55].

Angelakis et al. [56] searched for optical polarization plane rotations in emission of 10 radio-loud NLSy1 galaxies using a combined monitoring data set obtained by RoboPol and other monitoring programs. They concluded that all sources with multiple measurements show significant variability, both in EVPA and fractional polarization. However, due to either sparse sampling of EVPA curves or high level of noise, they could not confidently report the detection of a long $\left(>90^{\circ}\right)$ continuous EVPA rotation in NLSy1, despite having several candidates for such events. Long-term monitoring campaigns with more accurate observations are needed to provide clear evidence of similarity of this type of AGN to blazars in their optical polarization variability.

\section{Caveats with EVPA Ambiguity, Rotation Definition, and Hidden Rotations}

The EVPA defines orientation of the preferred polarization plane with respect to the celestial meridian. For this reason, the EVPA value is a factor of $180^{\circ}$ ambiguous, i.e., values EVPA $\pm n \times 180^{\circ}$ are mathematically equivalent. This means that every measurement of EVPA is, in fact, an infinite countable set of values separated by $180^{\circ}$, as is the difference between two measurements (see the left panel of Figure 7). The most natural way to solve this $180^{\circ}$ ambiguity is to assume that the variability time scale is much longer than the interval between measurements (see discussion in Section 3.3 of [45]) and minimize the difference of EVPA between each of the two consecutive measurements. Nevertheless, even the procedure of this minimization can be performed in several different ways. For instance, many authors minimize the following value $\Delta \theta_{n}=\left|\theta_{n}-\theta_{n-1}\right|-S L \sqrt{\sigma\left(\theta_{n}\right)^{2}+\sigma\left(\theta_{n-1}\right)^{2}}$, where $\theta_{n}$ and $\sigma\left(\theta_{n}\right)$ are $n$-th EVPA measurement and its uncertainty, $S L$ is the significance level typically set to unity. If $\Delta \theta_{n}>90^{\circ}$, the angle $\theta_{n}$ is shifted by $\pm n \times 180^{\circ}$, where the integer $\pm n$ is chosen in such a way that it minimizes $\Delta \theta_{n}$. If $\Delta \theta_{n} \leq 90^{\circ}$, then $\theta_{n}$ is left unchanged.

However, Kiehlmann et al. [26] assert that this method itself may lead to inconsistent results, since it depends on the choice of the initial interval. They suggest to exclude the term with uncertainties and use $\Delta \theta_{n}=\left|\theta_{n}-\theta_{n-1}\right|$ for minimization. Although, in general, these arguments are correct, we caution that this method of the $180^{\circ}$ ambiguity solution can lead to spurious $\sim 180^{\circ}$ jumps in the EVPA curve caused by measurements with high uncertainties. An example of such a case is shown in the right panel of Figure 7, where the black and blue EVPA curves are constructed with and without EVPA uncertainties taken into account. 

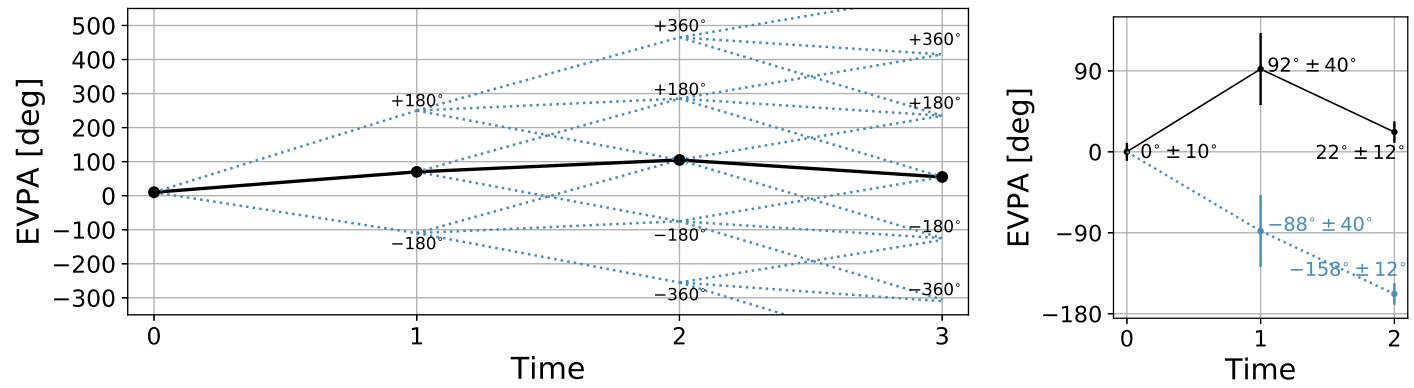

Figure 7. Left panel: an example of a family of EVPA curves demonstrating the $180^{\circ}$ ambiguity. All paths connecting consecutive points in the graph are mathematically equivalent. Only the three most probable escapes for each step are shown. Right panel: an example of a spurious jump in the EVPA curve introduced by the $180^{\circ}$ ambiguity solution method.

Besides the high sensitivity of EVPA curves to the particular procedure of the $180^{\circ}$ ambiguity solution and sampling, there is ambiguity in the EVPA rotation definition. Potentially, any significant change of EVPA between two measurements can be considered as an EVPA rotation. Usually, long amplitude, and smooth and monotonic changes of EVPA are of interest, and corresponding requirements are introduced into the definition of an EVPA rotation. For instance, our definition of an EVPA rotation stated that it must be comprised of at least four measurements and have a total amplitude $>90^{\circ}$, while the start and end points of a rotation event are defined by a change of the EVPA curve slope by a factor of $\geq 5$ or a change of its sign. In the cases with large gaps $\Delta t>30 \mathrm{~d}$ between consecutive measurements, we considered rotations being terminated at these gaps. A particular choice of the definition may affect parameters of derived EVPA rotations. A different approach for the identification of EVPA rotations was proposed by Larionov et al. [50], who suggest using the Discrete Correlation Function (DCF) between the $\mathrm{Q}$ and U Stokes parameters. This method, however, requires very well-sampled light curves that may be unavailable for monitoring programs with large samples and single observing facilities. For instance, the EVPA rotations detected by RoboPol are traced by 4 to 22 measurements, while the DCF method requires an order of magnitude with more dense sampling.

Studies of Angelakis et al. [41] and Hovatta et al. [43] demonstrate that HSP and ISP sources tend to have preferred directions of EVPA, which is presumably caused by an emission of a steady polarized source superimposed on the erratic component $[6,7,57]$. This component leads to an apparent shift of the measurements distribution centroid $Q_{c}, U_{c}$ from the origin of coordinates on the Q-U Stokes parameters plane. In such cases, rotations of the polarization plane may manifest themselves on the Q-U plane as consecutive loops around $\left(Q_{c}, U_{c}\right)$ instead of $(0,0)$. These events show a low-amplitude variability in EVPA curves and may be missed, since many studies identify rotations directly in the EVPA curves. Therefore, a significant fraction of EVPA rotations can be "hidden" and require analysis of the variability in the Q-U plane [58,59]. A handy tool for such analysis was proposed by Uemura et al. [60], who also demonstrated its efficiency using a combined set of Kanata and RoboPol observations [61].

\section{Future Prospects}

Despite the recent progress and advances provided by optical polarimetry monitoring programs, there are a number of questions related to polarization of blazars that remain unclear. We still do not know what process or processes produce polarization plane rotations in blazar emission and how they are related to $\gamma$-ray flares. The short-term polarization variability of AGN remains mostly unexplored. It is known that both fractional polarization and EVPA can significantly vary on the minutes-hours time scale $[50,53,62]$, but the origin of this variability is obscure. The solution of these and other problems requires continuation of systematic optical polarimetric monitoring programs accompanied by a multiwavelength total-flux follow-up. Moreover, the necessity of such programs is boosted in the 
context of future $X$-ray polarimetry missions $[63,64]$. The near-future $X$-ray polarimeters will require rather long integration times. Therefore, ongoing continuous monitoring of optical polarization in blazars will be extremely useful for picking highly polarized and active targets for observations in the X-rays. RoboPol results (see Section 3.4), as well as theoretical studies [65] suggest that at least HBL sources are expected to be highly polarized and variable in the X-rays band. Therefore, we expect a bright future for polarimetry of AGN in the optical in synergy with the X-rays [31].

Author Contributions: All authors contributed equally to the manuscript.

Funding: D.B. acknowledges support by the European Research Council (ERC) under the European Union's Horizon 2020 research and innovation program under the grant agreement No 771282.

Acknowledgments: The RoboPol project is a collaboration between Caltech in the USA, MPIfR in Germany, Torun Centre for Astronomy in Poland, the University of Crete/FORTH in Greece, and IUCAA in India.

Conflicts of Interest: The authors declare no conflict of interest.

\section{References}

1. Kinman, T.D.; Lamla, E.; Wirtanen, C.A. The optical brightness variations and polarization of the quasi-stellar radio source 3c 446. Astrophys. J. 1966, 146, 964. [CrossRef]

2. Angel, J.R.P.; Stockman, H.S. Optical and infrared polarization of active extragalactic objects. Annu. Rev. Astron. Astrophys. 1980, 18, 321. [CrossRef]

3. Moore, R.L.; Angel, J.R.P.; Duerr, R.; Lebofsky, M.J.; Wisniewski, W.Z.; Rieke, G.H.; Axon, D.J.; Bailey, J.; Hough, J.M.; McGraw, J.T. The noise of BL Lacertae. Astrophys. J. 1982, 260, 415-436. [CrossRef]

4. Brindle, C.; Hough, J.H.; Bailey, J.A.; Axon, D.J.; Schulz, H.; Kikuchi, S.; McGraw, J.T.; Wisniewski, W.J.; Fontaine, G.; Nadesu, D.; et al. Coordinated multisite observations of the variability of BL Lac. Mon. Not. R. Astron. Soc. 1985, 214, 619-638. [CrossRef]

5. Blinov, D.A.; Hagen-Thorn, V.A. Stochastic model of optical variability of BL Lacertae. Astron. Astrophys. 2009, 503, 103-106. [CrossRef]

6. Uemura, M.; Kawabata, K.S.; Sasada, M.; Ikejiri, Y.; Sakimoto, K.; Itoh, R.; Yamanaka, M.; Ohsugi, T.; Sato, S.; Kino, M. Bayesian approach to find a long-term trend in erratic polarization variations observed in blazars. Pub. Astron. Soc. Jpn. 2010, 62, 69. [CrossRef]

7. Villforth, C.; Nilsson, K.; Heidt, J.; Takalo, L.O.; Pursimo, T.; Berdyugin, A.; Lindfors, E.; Pasanen, M.; Winiarski, M.; Drozdz, M.; et al. Variability and stability in blazar jets on time-scales of years: Optical polarization monitoring of OJ 287 in 2005-2009. Mon. Not. R. Astron. Soc. 2010, 402, 2087-2111. [CrossRef]

8. Goldstein, J.; Samuel, J. A stochastic theory for the wavelength dependence of linear polarization of radio sources. Astrophys. J. 1967, 72, 799. [CrossRef]

9. Ledden, J.E.; Aller, H.D. The radio polarization of AO 0235+164-A rotating source. Astrophys. J. 1979, 229, L1-L3. [CrossRef]

10. Jones, T.W.; Rudnick, L.; Aller, H.D.; Aller, M.F.; Hodge, P.E.; Fiedler, R.L. Magnetic field structures in active compact radio sources. Astrophys. J. 1985, 290, 627-636. [CrossRef]

11. Kikuchi, S.; Inoue, M.; Mikami, Y.; Tabara, H.; Kato, T. A synchronous variation of polarization angle in OJ 287 in the optical and radio regions. Astron. Astrophys. 1988, 190, L8-L10.

12. Marscher, A.P.; Jorstad, S.G.; D'Arcangelo, F.D.; Smith, P.S.; Williams, G.G.; Larionov, V.M.; Oh, H.; Olmstead, A.R.; Aller, M.F.; Aller, H.D.; et al. The inner jet of an active galactic nucleus as revealed by a radio-to- $\gamma$-ray outburst. Nature 2008, 452, 966-969. [CrossRef]

13. Abdo, A.A.; Ackermann, M.; Ajello, M.; Axelsson, M.; Baldini, L.; Ballet, J.; Barbiellini, G.; Bastieri, D.; Baughman, B.M.; Bechtol, K.; et al. A change in the optical polarization associated with a $\gamma$-ray flare in the blazar 3C279. Nature 2010, 463, 919-923. [CrossRef]

14. Marscher, A.P.; Jorstad, S.G.; Larionov, V.M.; Aller, M.F.; Aller, H.D.; Lähteenmäki, A.; Agudo, I.; Smith, P.S.; Gurwell, M.; Hagen-Thorn, V.A.; et al. Probing the inner jet of the quasar PKS 1510-089 with multi-waveband monitoring during strong gamma-ray activity. Astrophys. J. 2010, 710, L126. [CrossRef]

15. Jorstad, S.; Marscher, A. The VLBA-BU-BLAZAR multi-wavelength monitoring program. Galaxies 2016, 4, 47. [CrossRef] 
16. Hagen-Thorn, V.A.; Larionov, V.M.; Blinov, D.A.; Arkharov, A.A.; Hagen-Thorn, E.I.; Borisova, E.V.; Takalo, L.O.; Sillanpää, A. Variability of the blazar 3C 454.3 during 2007-2010. Astron. Rep. 2013, 57, 726-733. [CrossRef]

17. Itoh, R.; Uemura, M.; Fukazawa, Y.; Kawabata, K. Polarimetric monitoring of jets with Kanata telescope. Galaxies 2018, 6, 16. [CrossRef]

18. Smith, P.S.; Montiel, E.; Rightley, S.; Turner, J.; Schmidt, G.D.; Jannuzi, B.T. Coordinated fermi/optical monitoring of blazars and the great 2009 september gamma-ray flare of 3C 454.3. Fermi symposium eConf proceedings C091122. arXiv 2009, arXiv:0912.5475 2009.

19. Agudo, I.; Molina, S.N.; Gómez, J.L.; Marscher, A.P.; Jorstad, S.G.; Heidt, J. Mapcat: Monitoring AGN with polarimetry at the calar alto telescopes. In International Journal of Modern Physics Conference Series; World Scientific Publishing Company: Singapore, 2012; Volume 8, pp. 299-302. [CrossRef]

20. Takalo, L.O.; Nilsson, K.; Lindfors, E.; Sillanpää, A.; Berdyugin, A.; Pasanen, M. Tuorla blazar monitoring program. AIP Conf. Proc. 2008, 1085, 705-707. [CrossRef]

21. Björnsson, C.I. Polarization properties of a source in relativistic motion. Astrophys. J. 1982, 260, 855-867. [CrossRef]

22. Konigl, A.; Choudhuri, A.R. A model of the polarization position-angle swings in BL Lacertae objects. Astrophys. J. 1985, 289, 188. [CrossRef]

23. Sillanpää, A.; Takalo, L.O.; Nilsson, K.; Kikuchi, S. Photopolarimetry of Bl-Lacertae. Astrophys. Space Sci. 1993, 206, 55-70. [CrossRef]

24. Larionov, V.M.; Jorstad, S.G.; Marscher, A.P.; Morozova, D.A.; Blinov, D.A.; Hagen-Thorn, V.A.; Konstantinova, T.S.; Kopatskaya, E.N.; Larionova, L.V.; Larionova, E.G.; et al. The outburst of the blazar S5 $0716+71$ in 2011 October: Shock in a helical jet. Astrophys. J. 2013, 768, 40. [CrossRef]

25. Marscher, A.P. Turbulent, extreme multi-zone model for simulating flux and polarization variability in blazars. Astrophys. J. 2014, 780, 87. [CrossRef]

26. Kiehlmann, S.; Savolainen, T.; Jorstad, S.G.; Sokolovsky, K.V.; Schinzel, F.K.; Marscher, A.P.; Larionov, V.M.; Agudo, I.; Akitaya, H.; Benítez, E.; et al. Polarization angle swings in blazars: The case of 3C 279. Astron. Astrophys. 2016, 590, A10. [CrossRef]

27. Zhang, H.; Chen, X.; Böttcher, M. Synchrotron polarization in blazars. Astrophys. J. 2014, 789, 66. [CrossRef]

28. Lyutikov, M.; Kravchenko, E.V. Polarization swings in blazars. Mon. Not. R. Astron. Soc. 2017, 467, 3876-3886. [CrossRef]

29. Nalewajko, K. A model of polarisation rotations in blazars from kink instabilities in relativistic jets. Galaxies 2017, 5, 64. [CrossRef]

30. Zhang, H.; Li, X.; Guo, F.; Giannios, D. Large-amplitude blazar polarization angle swing as a signature of magnetic reconnection. Astrophys. J. 2018, 862, L25. [CrossRef]

31. Peirson, A.L.; Romani, R.W. The polarization behavior of relativistic synchrotron jets. Astrophys. J. 2018, 864, 140. [CrossRef]

32. Clarke, D. Stellar Polarimetry; John Wiley \& Sons: Hoboken, NJ, USA, 2009. [CrossRef]

33. Boumis, P.; Steiakaki, A.; Mavromatakis, F.; Paterakis, G.; Papamastorakis, I. Seeing measurements at Skinakas observatory using the DIMM method. In Proceedings of the 5th Hellenic Astronomical Conference, Crete, Greece, 20-22 September 2001; p. 134.1.

34. Ramaprakash, A.N.; Rajarshi, C.V.; Das, H.K.; Khodade, P.; Modi, D.; Panopoulou, G.; Maharana, S.; Blinov, D.; Angelakis, E.; Casadio, C.; et al. RoboPol: A four-channel optical imaging polarimeter. Mon. Not. R. Astron. Soc. 2019, 485, 2355-2366. [CrossRef]

35. King, O.G.; Blinov, D.; Ramaprakash, A.N.; Myserlis, I.; Angelakis, E.; Baloković, M.; Feiler, R.; Fuhrmann, L.; Hovatta, T.; Khodade, P.; et al. The RoboPol pipeline and control system. Mon. Not. R. Astron. Soc. 2014, 442, 1706-1717. [CrossRef]

36. Nolan, P.L.; Abdo, A.A.; Ackermann, M.; Ajello, M.; Allafort, A.; Antolini, E.; Atwood, W.B.; Axelsson, M.; Baldini, L.; Ballet, J.; et al. Fermi large area telescope second source catalog. Astrophys. J. Suppl. Ser. 2012, 199, 31. [CrossRef]

37. Healey, S.E.; Romani, R.W.; Cotter, G.; Michelson, P.F.; Schlafly, E.F.; Readhead, A.C.; Giommi, P.; Chaty, S.; Grenier, I.A.; Weintraub, L.C. CGRaBS: An all-sky survey of gamma-ray blazar candidates. Astrophys. J. Suppl. Ser. 2008, 175, 97. [CrossRef] 
38. Richards, J.L.; Max-Moerbeck, W.; Pavlidou, V.; King, O.G.; Pearson, T.J.; Readhead, A.C.S.; Reeves, R.; Shepherd, M.C.; Stevenson, M.A.; Weintraub, L.C.; et al. Blazars in the fermi era: The OVRO $40 \mathrm{~m}$ telescope monitoring program. Astrophys. J. Suppl. Ser. 2011, 194, 29. [CrossRef]

39. Pavlidou, V.; Angelakis, E.; Myserlis, I.; Blinov, D.; King, O.G.; Papadakis, I.; Tassis, K.; Hovatta, T.; Pazderska, B.; Paleologou, E.; et al. The RoboPol optical polarization survey of gamma-ray-loud blazars. Mon. Not. R. Astron. Soc. 2014, 442, 1693-1705. [CrossRef]

40. Blinov, D.; Pavlidou, V.; Papadakis, I.E.; Hovatta, T.; Pearson, T.J.; Liodakis, I.; Panopoulou, G.V.; Angelakis, E.; Baloković, M.; Das, H.; et al. RoboPol: Optical polarization-plane rotations and flaring activity in blazars. Mon. Not. R. Astron. Soc. 2016, 457, 2252-2262. [CrossRef]

41. Angelakis, E.; Hovatta, T.; Blinov, D.; Pavlidou, V.; Kiehlmann, S.; Myserlis, I.; Böttcher, M.; Mao, P.; Panopoulou, G.V.; Liodakis, I.; et al. RoboPol: The optical polarization of gamma-ray-loud and gamma-ray-quiet blazars. Mon. Not. R. Astron. Soc. 2016, 463, 3365-3380. [CrossRef]

42. Itoh, R.; Nalewajko, K.; Fukazawa, Y.; Uemura, M.; Tanaka, Y.T.; Kawabata, K.S.; Madejski, G.M.; Schinzel, F.K.; Kanda, Y.; Shiki, K.; et al. Systematic study of gamma-ray-bright blazars with optical polarization and gamma-ray variability. Astrophys. J. 2016, 833, 77. [CrossRef]

43. Hovatta, T.; Lindfors, E.; Blinov, D.; Pavlidou, V.; Nilsson, K.; Kiehlmann, S.; Angelakis, E.; Fallah Ramazani, V.; Liodakis, I.; Myserlis, I.; et al. Optical polarization of high-energy BL Lacertae objects. Astron. Astrophys. 2016, 596, A78. [CrossRef]

44. Jermak, H.; Steele, I.A.; Lindfors, E.; Hovatta, T.; Nilsson, K.; Lamb, G.P.; Mundell, C.; Barres de Almeida, U.; Berdyugin, A.; Kadenius, V.; et al. The RINGO2 and DIPOL optical polarization catalogue of blazars. Mon. Not. R. Astron. Soc. 2016, 462, 4267-4299. [CrossRef]

45. Blinov, D.; Pavlidou, V.; Papadakis, I.; Kiehlmann, S.; Panopoulou, G.; Liodakis, I.; King, O.G.; Angelakis, E.; Baloković, M.; Das, H.; et al. RoboPol: First season rotations of optical polarization plane in blazars. Mon. Not. R. Astron. Soc. 2015, 453, 1669-1683. [CrossRef]

46. Blinov, D.; Pavlidou, V.; Papadakis, I.; Kiehlmann, S.; Liodakis, I.; Panopoulou, G.V.; Angelakis, E.; Baloković, M.; Hovatta, T.; King, O.G.; et al. RoboPol: Connection between optical polarization plane rotations and gamma-ray flares in blazars. Mon. Not. R. Astron. Soc. 2018, 474, 1296-1306. [CrossRef]

47. Blinov, D.; Pavlidou, V.; Papadakis, I.; Kiehlmann, S.; Liodakis, I.; Panopoulou, G.V.; Pearson, T.J.; Angelakis, E.; Baloković, M.; Hovatta, T.; et al. RoboPol: Do optical polarization rotations occur in all blazars? Mon. Not. R. Astron. Soc. 2016, 462, 1775-1785. [CrossRef]

48. Hovatta, T.; Pavlidou, V.; King, O.G.; Mahabal, A.; Sesar, B.; Dancikova, R.; Djorgovski, S.G.; Drake, A.; Laher, R.; Levitan, D. Connection between optical and $\gamma$-ray variability in blazars. Mon. Not. R. Astron. Soc. 2014, 439, 690-702. [CrossRef]

49. Kiehlmann, S.; Blinov, D.; Pearson, T.J.; Liodakis, I. Optical EVPA rotations in blazars: Testing a stochastic variability model with RoboPol data. Mon. Not. R. Astron. Soc. 2017, 472, 3589-3604. [CrossRef]

50. Larionov, V.; Jorstad, S.; Marscher, A.; Smith, P. Polarization vector rotations: Real, spurious, hidden and imaginary. Galaxies 2016, 4, 43. [CrossRef]

51. Foschini, L.; Berton, M.; Caccianiga, A.; Ciroi, S.; Cracco, V.; Peterson, B.M.; Angelakis, E.; Braito, V.; Fuhrmann, L.; Gallo, L. Properties of flat-spectrum radio-loud narrow-line Seyfert 1 galaxies. Astron. Astrophys. 2015, 575, A13. [CrossRef]

52. Paliya, V.S.; Ajello, M.; Rakshit, S.; Mandal, A.K.; Stalin, C.S.; Kaur, A.; Hartmann, D. Gamma-ray-emitting narrow-line seyfert 1 galaxies in the sloan digital sky survey. Astrophys. J. 2018, 853, L2. [CrossRef]

53. Itoh, R.; Tanaka, Y.T.; Fukazawa, Y.; Kawabata, K.S.; Kawaguchi, K.; Moritani, Y.; Takaki, K.; Ueno, I.; Uemura, M.; Akitaya, H. Minute-scale rapid variability of the optical polarization in the narrow-line seyfert 1 galaxy PMN J0948+0022. Astrophys. J. 2013, 775, L26. [CrossRef]

54. Eggen, J.R.; Miller, H.R.; Maune, J.D. The variable optical polarization and fermi observations of PMN J0948+0022. Astrophys. J. 2013, 773, 85. [CrossRef]

55. Maune, J.D.; Eggen, J.R.; Miller, H.R.; Marshall, K.; Readhead, A.C.S.; Hovatta, T.; King, O. The extreme behavior of the radio-loud narrow-line Seyfert 1 galaxy J0849+5108. Astrophys. J. 2014, 794, 93. [CrossRef]

56. Angelakis, E.; Kiehlmann, S.; Myserlis, I.; Blinov, D.; Eggen, J.; Itoh, R.; Marchili, N.; Zensus, J.A. Optical polarisation variability of radio-loud narrow-line Seyfert 1 galaxies. Search for long rotations of the polarisation plane. Astron. Astrophys. 2018, 618, A92. [CrossRef] 
57. Hagen-Thorn, V.A.; Larionova, E.G.; Jorstad, S.G.; Björnsson, C.I.; Larionov, V.M. Analysis of the long-term polarization behaviour of BL Lac. Astron. Astrophys. 2002, 385, 55-61. [CrossRef]

58. Ikejiri, Y.; Uemura, M.; Sasada, M.; Ito, R.; Yamanaka, M.; Sakimoto, K.; Arai, A.; Fukazawa, Y.; Ohsugi, T.; Kawabata, K.S.; et al. Photopolarimetric monitoring of blazars in the optical and near-infrared bands with the Kanata telescope. I. Correlations between flux, color, and polarization. Pub. Astron. Soc. Jpn. 2011, 63, 639-675. [CrossRef]

59. Larionov, V.M.; Villata, M.; Raiteri, C.M.; Jorstad, S.G.; Marscher, A.P.; Agudo, I.; Smith, P.S.; Acosta-Pulido, J.A.; Árévalo, M.J.; Arkharov, A.A. Exceptional outburst of the blazar CTA 102 in 2012: The GASP-WEBT campaign and its extension. Mon. Not. R. Astron. Soc. 2016, 461, 3047-3056. [CrossRef]

60. Uemura, M.; Itoh, R.; Xu, L.; Nakayama, M.; Wu, H.Y.; Watanabe, K.; Takahashi, S.; Fujishiro, I. TimeTubes: Visualization of polarization variations in blazars. Galaxies 2016, 4, 23. [CrossRef]

61. Uemura, M.; Itoh, R.; Liodakis, I.; Blinov, D.; Nakayama, M.; Xu, L.; Sawada, N.; Wu, H.Y.; Fujishiro, I. Optical polarization variations in the blazar PKS 1749+096. Pub. Astron. Soc. Jpn. 2017, 69, 96. [CrossRef]

62. Itoh, R.; Fukazawa, Y.; Tanaka, Y.T.; Abe, Y.; Akitaya, H.; Arai, A.; Hayashi, M.; Hori, T.; Isogai, M.; Izumiura, H.; et al. Dense optical and near-infrared monitoring of CTA 102 during high state in 2012 with OISTER: Detection of intra-night "orphan polarized flux flare". Astrophys. J. 2013, 768, L24. [CrossRef]

63. Fabiani, S. Instrumentation and future missions in the upcoming era of X-ray polarimetry. Galaxies 2018, 6, 54. [CrossRef]

64. Weisskopf, M. An overview of X-ray polarimetry of astronomical sources. Galaxies 2018, 6, 33. [CrossRef]

65. Zhang, H.; Böttcher, M. X-ray and gamma-ray polarization in leptonic and hadronic jet models of blazars. Astrophys. J. 2013, 774, 18. [CrossRef]

(C) 2019 by the authors. Licensee MDPI, Basel, Switzerland. This article is an open access article distributed under the terms and conditions of the Creative Commons Attribution (CC BY) license (http:/ / creativecommons.org/licenses/by/4.0/). 